\title{
Hot-electron photocurrent detection of near-infrared light based on $\mathrm{ZnO}$ Y. Hou, ${ }^{1,2, a)}$ H. Liang, ${ }^{1,3}$ A. Tang, ${ }^{3}$ X. Du, ${ }^{1,3}$ and Z. Mei ${ }^{1,3, b)}$ \\ ${ }^{1)}$ Songshan Lake Materials Laboratory, Dongguan, Guangdong 523808, China \\ ${ }^{2}$ School of microelectronics, Shandong University, No. 1500 Shunhua Road, Lixia District, Jinan, Shandong Province, 250101, China \\ 3) Key Laboratory for Renewable Energy, Beijing National Laboratory for Condensed Matter Physics, Institute of Physics, Chinese Academy of Sciences, Beijing 100190, China \\ (Dated: 7 January 2021)
}

We demonstrate an unconventional near infrared photodetector fabricated from a ZnO chip with a metallic subwavelength grating structure as contact and optical window, which harvests hot electrons generated by plasmonic resonances introduced by incident light. The grating structure has a strong selection of the polarization of incident light, meaning that the detector is naturally polarization-sensitive. In our device, the polarization extinction ratio is as high as 64:1, much higher than that relying on crystal orientations. Since the photoresponse is introduced by plasmonic resonance, a narrow photoresponse spectrum with a linewidth of $32.1 \mathrm{~nm}$ at $1.201 \mu \mathrm{m}$ is obtained. By simply changing the grating period, the spectral position can be tailored freely within the near infrared region, i.e., wavelength-selective. Such a spectral response is not likely to be realized with conventional semiconductor photodetectors, which depend on the band edge absorption. We propose a modified Fowler's model, which well explains the line shape of photoresponse spectra of such devices.

Near infrared (NIR) photodetectors (PDs) are widely applied in environmental surveillance, non-invasive therapies, food inspections and astronomy. ${ }^{1-4}$ Highly efficient NIR-PDs are normally fabricated from narrow band gap semiconductors and pyroelectric materials. ${ }^{5-7}$ Limited by the working mechanisms (photon absorption by either band edge absorption or thermally driving dipole moment realignment), the photoresponse spectra of such PDs are broadband. In order to achieve spectrally selective narrow-band NIR-PDs, optical filters have to be employed, which makes the detecting system bulky and less sensitive. In addition, such PDs are immune to polarization, which plays a pivotal role in photobiology and optogenetics. ${ }^{8,9}$ On the other hand, $\mathrm{ZnO}$ is widely used for photonic devices including optical biosensors and photodetectors owning to its outstanding optical properties. ${ }^{10}$ However, the working wavelengths of $\mathrm{ZnO}$ based devices are limited to UV band due to its large band gap $(3.37 \mathrm{eV})$. Many work have been dedicated to extending the photoresponse to IR region, ${ }^{11-13}$ which is likely to inspire new optical detection platforms, since direct fabrication of NIR-PDs on $\mathrm{ZnO}$ is not possible due to the large band gap. The rapid development of the research on surface plasmon induced hot-electron transfer in recent years provides a promising solution to this issue. ${ }^{14,15}$ Different from the traditional NIR-PDs as mentioned above, surface plasmon induced hot-electron transfer is a result of the resonance between the surface electrons of metallic nanostructures and incident photons. The peak position and linewidth can be tuned by modifying the dimensions of plasmonic nanostructures, thus realizing narrow-band spectral-selective PDs on narrow band gap semiconductors. ${ }^{16,17}$ It is worth to point out that the lifetime of hot electrons is extremely short, where the Auger scatter-

\footnotetext{
a) Author's current address: Optoelectronics Research Centre, the University of Southampton,UK

${ }^{b}$ Authors to whom correspondence should be addressed: Yaonan Hou, houyaonan@gmail.com; Zengxia Mei, zxmei@iphy.ac.cn
}

ing followed by thermal relaxation processes accomplishes in less than 1 ps. ${ }^{15}$ Therefore, the hot electrons must be collected (i.e., charge transfer) on the same time scale. While $\mathrm{ZnO}$ has been demonstrated as a good candidate for electron collectors in many devices such as dye-sensitized solar cells and colloidal quantum dot PDs, ${ }^{18,19}$ the research on $\mathrm{ZnO}$ plasmonic NIR-PDs is still in its infancy. ${ }^{13,20}$

In previous work, Pescaglini et.al. reported a Au nanorod coated $\mathrm{ZnO}$ nanowire system with NIR photoresponse. ${ }^{13}$ This structure is subjected to surface states and random orientations of the Au nanorods, which limits the device performance. In this work, we chose metallic subwavelength gratings as the plasmonic architecture. The structure has been proved powerful to generate infrared plasmonic resonances on $\mathrm{ZnO} .^{21} \mathrm{By}$ simply changing the grating period $(\Lambda)$, the resonance peak can be tuned from $1 \mu \mathrm{m}$ to $10 \mu \mathrm{m} .{ }^{21}$ Moreover, the resonance depends on the polarization of the incident light, implying polarization-sensitive PDs are likely to be fabricated. ${ }^{21}$ In this letter, we present a room-temperature working ZnO NIR-PD with a planar Schottky contact fabricated from $50 \mathrm{~nm}$ thick Au gratings, with a period of $800 \mathrm{~nm}(\Lambda=800 \mathrm{~nm})$ and a gap of $150 \mathrm{~nm}(a=150 \mathrm{~nm})$. Together with $\mathrm{ZnO}$ under illumination, plasmonic resonances will be introduced to generate hot-electron photocurrent. The device exhibits a strong photoresponse at $1.2 \mu \mathrm{m}$ with a narrow line width of $32.1 \mathrm{~nm}$ and an extremely low dark current bellow $10 \mathrm{fA}$. The photoresponse exhibits a strong polarization sensitivity with an extinction ratio up to $64: 1$, a record-high value of $\mathrm{ZnO}$ polarization photodetectors. Over adjusting the periodicity of the gratings, we demonstrate that the peak of photoresponse spectra can be adjusted from $1 \mu \mathrm{m}$ to $1.3 \mu \mathrm{m}$. Most importantly, we propose a modified Fowler's model, which well interprets the photoresponse spectra. This work provides an approach to exploring $\mathrm{ZnO}$ plasmonics in the infrared region.

Fig.1a schematically shows the device architecture. The device is fabricated from a $350 \mathrm{~nm}$ epitaxial $\mathrm{ZnO}$ film on sapphire substrate by molecular-beam epitaxy (MBE). In order to improve the conductivity through defect control, ${ }^{22,23}$ n-type 
$\mathrm{ZnO}$ was grown by $\mathrm{Ga}$ doping, with a carrier concentration of $9.878 \times 10^{17} / \mathrm{cm}^{3}$ and carrier mobility of $17.45 \mathrm{~cm}^{2} /(\mathrm{V} \cdot \mathrm{s})$, measured by Van der Pauw method. The hot-electron photocurrent is normally much lower than that generated from near band gap absorption. ${ }^{14,17} \mathrm{~A}$ moderate doped sample was selected to expect a high signal to noise ratio, because high doping is always associated with a high darkcurrent. ${ }^{24} \mathrm{Be}$ fore contact fabrication, oxygen plasma was used to perform the surface treatment. Then $2 \mathrm{~nm} \mathrm{SiO}_{2}$ passivation layer was deposited by electron-beam evaporation with a pressure of $2 \times 10^{-6} \mathrm{mbar}$ and a deposition rate of $0.1 \mathrm{~nm} / \mathrm{s}$. Atop the film, $1 \mathrm{~nm}$ Ti and $50 \mathrm{~nm}$ Au were deposited by thermal evaporation (pressure of $2 \times 10^{-6}$ mbar and deposition speed of $0.1 \mathrm{~nm} / \mathrm{s}$ ) in sequence as Schottky contact. Optical active area with grating structure was fabricated by focused ion beam milling. Fig.1b shows the top-view of the grating $(\Lambda=800 \mathrm{~nm}$ and $a=150 \mathrm{~nm}$ ) by scanning electron microscope (SEM) Simulation was firstly performed using COMSOL under periodic boundary condition to have a preliminary insight into the optical properties, where $\mathrm{ZnO}$ refractive index of 1.935 was adopted ${ }^{25}$ Fig. 1c shows the simulated absorption spectra of the Au grating- $\mathrm{ZnO}$ system under incident light with in-plane polarization orientations perpendicular and parallel to the gratings, respectively labelled as "x-pol." (polarization along $\mathrm{x}$-axis) and "y-pol."(polarization along y-axis), according to the coordinate system shown in Fig.1a. Under x-pol. illumination, a weak symmetric absorption can be observed at $1.24 \mu \mathrm{m}$, whilst an asymmetric strong absorption peak at $1.2 \mu \mathrm{m}$ appears under y-pol. illumination as a result of plasmonic resonances. Electric field profiles were plotted in Fig. 1d to have a further insight. In Fig.1d, light irradiates from the top in both cases. Under x-pol. illumination(Fig.1d left), clear transmitted modes with a small part of surface coupled light can be observed. Therefore, the resonance strength is weak. In contrast, evanescent electric field is formed under y-pol. illumination (Fig.1d right), introducing strong plasmonic resonances in the gap of the gratings. In addition, the transmitted optical modes form standing wave in the bottom of the Au surface, which will further enhance the absorption. Since our structures are on subwavelength scale, the asymmetric shape of the absorption spectrum under y-pol. illumination could be related with the Fano resonances, which is the single gap resonance buried in the resonances of the entire grating structure. After fabrication, the electrical properties of our device were characterized by a Keithley 4200 semiconductor analyzer with a current resolution down to $f A$. Spectral response was measured with a illumination from a $150 \mathrm{~W}$ Xenon lamp followed by a monochromator with a focus length of $0.5 \mathrm{~m}$, and photocurrent was recorded by the Keithley 4200 Polarization-dependent characteristics were implemented by placing a broadband polarizer in front of the device. Rotation angle of the polarizer with respect to the grating orientations as well as the photoresponse were recorded. Transient response was measured by using an oscilloscope under pulsed light illumination.

Fig.2a shows the current as a function of applied voltage (IV curve) measured under dark and polarized-light illuminations. As shown in the figure, the dark current is as low as

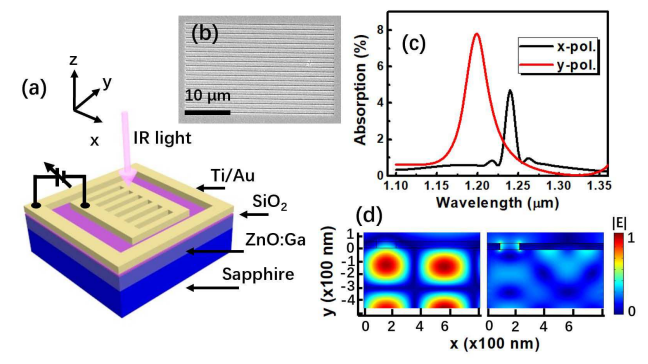

FIG. 1. a. Schematic configuration of our device; b. SEM top view of the gratings with $\Lambda=800 \mathrm{~nm}$ and $a=150 \mathrm{~nm}$ (scale bar=10 $\mu \mathrm{m})$; c. simulated absorption spectra under x-pol. and y-pol. incidence; d. cross-sectional electric field profile of the Au grating- $\mathrm{ZnO}$ system (left: under $\mathrm{x}$-pol. illumination, right: under $\mathrm{y}$-pol. illumination).

$10 \mathrm{fA}$ at $3 \mathrm{~V}$, indicating an ultra-low leakage due to surface passivation. Under x-pol. and y-pol. illumination, $4 p A$ and $0.28 n A$ photocurrents are observed, respectively. The signal to noise ratio is $2.8 \times 10^{4}: 400: 1\left(I_{y-p o l .}: I_{x-\text { pol. }}: I_{\text {dark }}\right)$. The result indicates that the device is sensitive to the in-plane polarization of the incident light; and the y-pol. photoresponse dominates the device performance. The mechanism of photoresponse to the polarized NIR light of our device is depicted by Fig.2b, where the photo-generated electron undergoes 4 steps including generation and circulation as labelled in the figure. Under NIR illumination, the plasmonic resonances are excited depending on the polarization and wavelength (y-pol. light labelled as a red arrow and a cross-in-circle; and x-pol. light labelled as a blue arrow and an arrowed-in-circle at step 1). Then, the hot-electrons generated by the plasmonic resonances with sufficient energy are collected by the Schottky barrier at step 2. After this, the charge balance is broken at both $\mathrm{Au}$ and $\mathrm{ZnO}$ sides, where the electrons are collected by the counter electrodes on $\mathrm{ZnO}$ (step 3). Under external bias, the electron will circulate back to the working electrode to form photocurrent in the system (step 4). It is worth to mention that in step 2, we have a $2 \mathrm{~nm} \mathrm{SiO}_{2}$ passivation layer on $\mathrm{ZnO}$ surface. The electron avalanche is likely to happen in this layer, which offers us the eventual large hot-electron response.

Spectral photoresponse under both polarization is plotted in Fig. 3a. A dominant narrow-band response with a peak at $1.201 \mu \mathrm{m}$ is observed under $\mathrm{y}$-pol. illumination, with a photoresponsivity of $8.36 \mu \mathrm{A} / \mathrm{W}$ and a full width at halfmaximum (FWHM) of $32.1 \mathrm{~nm}$. In contrast, under x-pol. illumination, the peak red-shifts to $1.238 \mu \mathrm{m}$, with the photoresponsivity of $1.54 \times 10^{-2} \mu \mathrm{A} / \mathrm{W}$ and FWHM of $25 \mathrm{~nm}$. The nature of narrow-band photoresponse of our device is fundamentally different from the near band-gap absorption in $\mathrm{ZnO}$ UV photodetectors, where the latter leads to a broad-band photoresponse. ${ }^{26}$ In order to quantitatively study the spectral photoresponse of plasmonic hot-electrons, many previous work just fitted their results into $I \approx C_{F} \frac{\left(h v-q \phi_{b}\right)^{2}}{h v}$ by following 


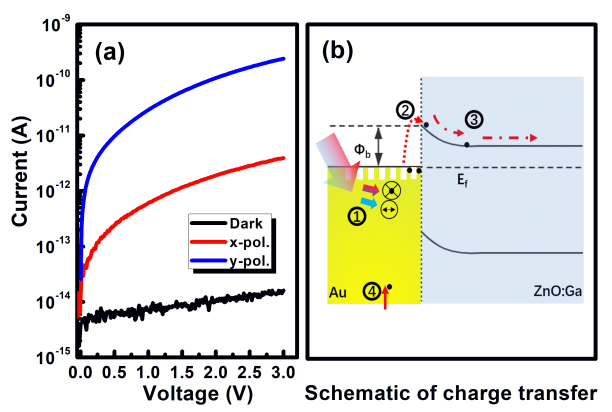

FIG. 2. a. IV curve of the device under x-pol., y-pol. illumination and dark; b. Schematic of the device working mechanism.

Fowler's early work, ${ }^{13,14,17,27-30}$ where $C_{F}$ is the hot-electron emission coefficient, $h v$ the incident photon energy and $q \phi_{b}$ the Schottky barrier height. However, it is known that the plasmonic resonant frequency heavily depends on the dimension and geometry of the structures utilised. Therefore, this equation is not always successfully to fit the spectral response, e.g., in our case. To illustrate the photoresponse, we propose a modified Fowler's model,

$$
\begin{aligned}
R & =G(f) \times C_{F} \frac{\left(h v-q \phi_{b}\right)^{2}}{h v} \\
& =C_{0} \operatorname{sinc}^{2}\left(\frac{a \pi}{\lambda}\right) \times \frac{\left(h v-q \phi_{b}\right)^{2}}{h v} .
\end{aligned}
$$

where $G(f)$ is a resonant function related with the device

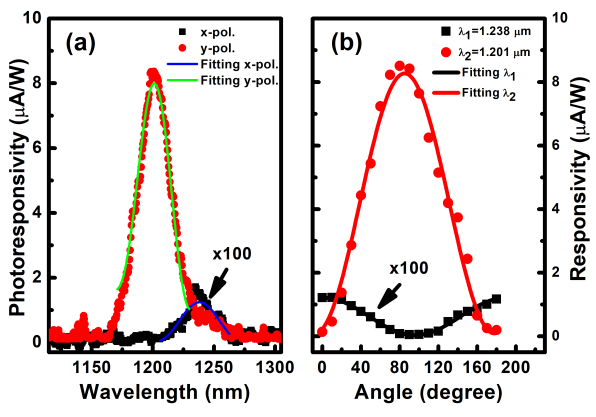

FIG. 3. a. Spectral responsivity of the photodetector under x-pol.(black-dotted curve) and y-pol.(red-dotted curved) illumination; where the solid lines are fitting curves using modified Fowler's model; b. Photoresponsivity as a function of polarization at the resonant peaks 1.201 and $1.238 \mu \mathrm{m}$, respectively.

geometry, which is expressed as a diffraction function determined by the gap of grating, $a$ (the first term in second line) ${ }^{31} \lambda$ is wavelength; and $C_{0}$ is a constant. As one can see, our experimental results can be well fitted into equation (1). We consider that the modified Fowler's model in this work is applicable to calculate the spectral photoresponse in gratinglike plasmonic nanostructures. Since the photodetector is a narrow-band, it is better to define a figure of merit (FoM) to characterize its optoelectronic properties (i.e., linewidth and photoresponsivity). We adopted the following equation,

$$
F o M=Q \times R
$$

where $Q$ is Q-factor calculated from $Q=\frac{\lambda_{p}}{\delta \lambda}$, with $\lambda_{p}$ and $\delta \lambda$ the responsivity peak and FWHM; and $R$ the value of photoresponsivity. From equation (2), the FoM at the two resonant peaks are obtained 312.8 (under y-pol.) and 0.76 (under $\mathrm{x}$-pol.), respectively.

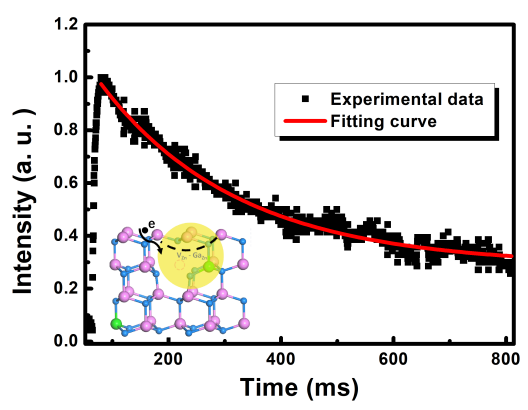

FIG. 4. Decay trace of the photocurrent (black squares), and the fitting curve (red); inset: schematic figure of the $\left(V_{Z n}-G a_{Z n}\right)^{-1}$ defect complex.

The sensitivity to the polarization is further measured under illumination as shown in Fig.3b, where the photoresponsivity is plotted as a function of rotation angles of polarizer $(\theta)$. The periodic varying photoresponsivity can be fitted by Malus's law, $R(\theta)=R_{0} \cos ^{2}(\theta)$ with a $\pi$-period, where $R_{0}$ is the peak photoresponsivity. Clearly, there's a phase difference of $\pi$ between the two resonant peaks, agreeing with the simulation results shown in Fig.1c-d. Furthermore, we observed that the extinction ratio (calculated from $R_{\max } / R_{\min }$ in Fig.3b) under $\mathrm{x}$-pol. (y-pol.) illumination is 64 (25), much higher than those devices relying on the crystal orientations. ${ }^{32}$

The transient photoresponse was studied with an oscilloscope to record the photoresponsivity generated by pulsed illumination. As shown in Fig. 4, a slow decay trace is observed when the light if off. The decay trace can be fitted into a single exponential decay, $I(t)=I_{0} \exp \left(-\frac{t}{\tau_{0}}\right)$, where $I_{0}$ and $\tau_{0}$ are peak photocurrent and decay lifetime, respectively. The decay lifetime is $245 \mathrm{~ms}$ from the fitting result. The slow decay (also named as persistent photocurrent, PPC) was also observed in the inter-band transition UV PDs, where a fast decay is normally observable when the light is turned off, due to the recombination of photo-excited carriers. Following this, 


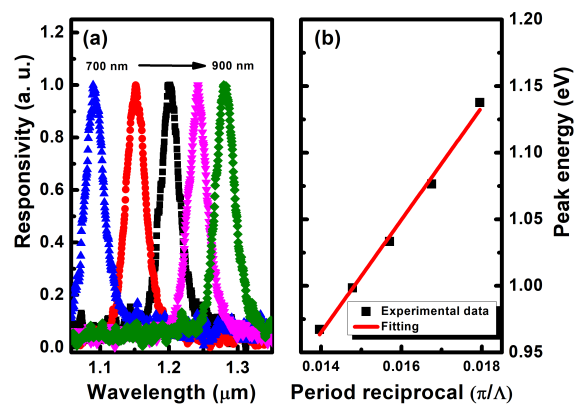

FIG. 5. a. Spectral photoresponse of the gratings with $\Lambda$ increasing from 700 to $900 \mathrm{~nm}$ with a step of $50 \mathrm{~nm}$; b. The resonant peak energy as a function of reciprocal of $\Lambda$.

the slow decay often presents with a lifetime as long as seconds, where it is ascribed to surface states, oxygen vacancies and so on. ${ }^{33}$ Obviously, our device is different from UV PDs in both the decay trace and the working mechanism. To explain the decay lifetime, we firstly ruled out the carrier recombination lifetime of $16 \mathrm{~ns},{ }^{34}$ and transit time between electrodes $(0.38 \mu \mathrm{s})$. Moreover, the density of oxygen vacancies of such samples is suppressed as demonstrated in our previous study. ${ }^{22}$ In this work, we attributed it to the defect complex $\left(V_{Z n}-G a_{Z n}\right)^{-1}$ near the interface, which is a deep level defect locating $1.085 \mathrm{eV}$ above the valence band maximum. ${ }^{34}$ The naturally formed one electron charge (denoted as " -1 ") will form a local barrier to electrons surrounding the defect (inset of Fig. 4). Under illumination, such defects will be neutralized by the photo-generated holes, lowering the local barrier. When the light is off, the negative charge restores, hindering the transportation of residual electrons, which need to travel to the working electrode to form a charge-balanced system. Although PPC slows down the transient response, it is worth to point out it could contribute to photocurrent gain. ${ }^{35}$ This can be interpreted by the enhanced carrier life time, because carrier recombination is obstructed in our case. Finally, we demonstrate that our NIR PD is able to select the photoresponse spectra in analogy to the band gap engineering, ${ }^{36-39}$ but without alloying extra elements with the $\mathrm{ZnO}$. Since the resonant peak is strongly related with the grating period $(\Lambda),{ }^{21}$ we fabricated a series of samples with $\Lambda$ ranging from 800 to $900 \mathrm{~nm}$. The photoresponse spectra under y-pol. illumination are shown in Fig.5a. Clearly, blue shift can be observed by decreasing $\Lambda$, and vice versa. By just changing the grating period from 800 to $900 \mathrm{~nm}$, the photoresponse peak can be continually tuned from 1.1 to $1.3 \mu \mathrm{m}$ without band gap engineering. Fig. 5b shows the peak energy as a function of the grating period. Clearly, the energy of the resonant peak are linearly increasing with the reciprocal of $\Lambda$, with a slope of 1.3657. This result provides us a guidance for fabricating subwavelength gratings with desired resonant peak.

In summary, we demonstrated a NIR PD with designated photoresponse at $1.2 \mu \mathrm{m}$ with subwavelength grating structures fabricated on $\mathrm{ZnO}$, based on the hot-electron transfer induced by surface plasmon resonances. The device is sensitive to the polarization of incident light, with an extinction ratio up to 64 under y-pol. illumination and photoresponsivity of $8.36 \mu \mathrm{A} / \mathrm{W}$. The photoresponse spectra show a narrow linewidth down to $25 \mathrm{~nm}$, different from band gap absorption spectrum. To explain this, we introduced a modified Fowler's model. Over changing the period of the gratings, the photoresponse spectra can be tuned accordingly, realizing the spectral selection without employing band gap engineering. We also investigated the transient response of our device, which is limited by the material rather than the hot-electron collection process. Further improvements of materials and device design would give rise to a faster photoresponse. Overall, our findings provide important knowledge to hot-electron devices, especially useful for developing high-performance and functional NIR PDs on group II-oxides semiconductors.

Acknowledgements This research is supported by National Natural Science Foundation of China (Grants No. 11675280, 11674405, 61874139, 11875088, and 61904201); and Qilu Yong Scholarship Program, Shandong University, China. Y.H acknowledges Optoelectronics Research Centre, the University of Southampton, for accessing to the COMSOL software.

DATA AVAILABILITY The data that support the findings of this study are available from the corresponding author upon reasonable request.

${ }^{1}$ K. Zhong, Y. He, L. Deng, X. Yan, X. Li, Y. Tang, S. Hou, and L. Tang, "A near-infrared fluorescent probe for h2s based on tandem reaction to construct iminocoumarin-benzothiazole and its application in food, water, living cells," Analytica Chimica Acta (2020).

${ }^{2}$ H. W. Siesler, Y. Ozaki, S. Kawata, and H. M. Heise, Near-infrared spectroscopy: principles, instruments, applications (John Wiley \& Sons, 2008). ${ }^{3}$ J. Li, B. Li, J. Sun, C. Ma, S. Wan, Y. Li, R. Göstl, A. Herrmann, K. Liu, and H. Zhang, "Engineered near-infrared fluorescent protein assemblies for robust bioimaging and therapeutic applications," Advanced Materials 32, 2000964 (2020).

${ }^{4}$ H. Umehata, I. Smail, A. Swinbank, K. Kohno, Y. Tamura, T. Wang, Y. Ao, B. Hatsukade, M. Kubo, K. Nakanishi, et al., "Alma deep field in ssa22-a near-infrared-dark submillimeter galaxy at $z=4.0$," Astronomy \& Astrophysics 640, L8 (2020)

${ }^{5}$ A. D. Stiff-Roberts, "Quantum-dot infrared photodetectors: a review," Journal of Nanophotonics 3, 031607 (2009).

${ }^{6} \mathrm{C}$. L. Tan and H. Mohseni, "Emerging technologies for high performance infrared detectors," Nanophotonics 7, 169-197 (2018).

B. Mukherjee, Y. Cai, H. R. Tan, Y. P. Feng, E. S. Tok, and C. H. Sow, "Nir schottky photodetectors based on individual single-crystalline gese nanosheet," ACS applied materials \& interfaces 5, 9594-9604 (2013).

${ }^{8}$ M. L. Harter, I. C. Felkner, W. W. Mantulin, D. L. McInturff, J. N. Marx, and P.-S. Song, "Excited states and photobiological properties of potential dna cross-linking agents, the benzodipyrones," Photochemistry and Photobiology 20, 407-413 (1974).

M. Wang, F. He, H. Li, S. Yang, J. Zhang, P. Ghosh, H.-H. Wang, and Z. Nie, "Near-infrared light-activated dna-agonist nanodevice for nongenetically and remotely controlled cellular signaling and behaviors in live animals," Nano letters 19, 2603-2613 (2019).

${ }^{10}$ A. Tereshchenko, M. Bechelany, R. Viter, V. Khranovskyy, V. Smyntyna, N. Starodub, and R. Yakimova, "Optical biosensors based on zno nanostructures: advantages and perspectives. a review," Sensors and Actuators B: tructures: advantages and persp
Chemical 229, 664-677 (2016)

${ }^{11}$ Y. E. Kesim, E. Battal, and A. K. Okyay, "Plasmonic materials based on zno films and their potential for developing broadband middle-infrared absorbers," AIP Advances 4, 077106 (2014). 
${ }^{12}$ C. Samanta, S. Bhattacharya, A. K. Raychaudhuri, and B. Ghosh, "Broadband (ultraviolet to near-infrared) photodetector fabricated in n-zno/p-s nanowires core-shell arrays with ligand-free plasmonic au nanoparticles," The Journal of Physical Chemistry C 124, 22235-22243 (2020).

${ }^{13}$ A. Pescaglini, A. Martín, D. Cammi, G. Juska, C. Ronning, E. Pelucchi, and D. Iacopino, "Hot-electron injection in au nanorod-zno nanowire hybrid device for near-infrared photodetection," Nano letters 14, 6202-6209 (2014)

${ }^{14}$ M. W. Knight, H. Sobhani, P. Nordlander, and N. J. Halas, "Photodetection with active optical antennas," Science 332, 702-704 (2011).

${ }^{15} \mathrm{C}$. Clavero, "Plasmon-induced hot-electron generation at nanoparticle/metal-oxide interfaces for photovoltaic and photocatalytic devices," Nature Photonics 8, 95-103 (2014)

${ }^{16}$ A. Sobhani, M. W. Knight, Y. Wang, B. Zheng, N. S. King, L. V. Brown, Z. Fang, P. Nordlander, and N. J. Halas, "Narrowband photodetection in the near-infrared with a plasmon-induced hot electron device," Nature communications 4, 1-6 (2013).

${ }^{17}$ M. W. Knight, Y. Wang, A. S. Urban, A. Sobhani, B. Y. Zheng, P. Nordlander, and N. J. Halas, "Embedding plasmonic nanostructure diodes enhances hot electron emission," Nano letters 13, 1687-1692 (2013).

${ }^{18}$ J. A. Anta, E. Guillén, and R. Tena-Zaera, "Zno-based dye-sensitized solar cells," The Journal of Physical Chemistry C 116, 11413-11425 (2012).

${ }^{19}$ H. Wang, V. Gonzalez-Pedro, T. Kubo, F. Fabregat-Santiago, J. Bisquert, Y. Sanehira, J. Nakazaki, and H. Segawa, "Enhanced carrier transport distance in colloidal pbs quantum-dot-based solar cells using zno nanowires," The Journal of Physical Chemistry C 119, 27265-27274 (2015).

${ }^{20}$ T. D. Dao, S. Ishii, T. Yokoyama, T. Sawada, R. P. Sugavaneshwar, K. Chen, Y. Wada, T. Nabatame, and T. Nagao, "Hole array perfect absorbers for spectrally selective midwavelength infrared pyroelectric detectors," Acs Photonics 3, 1271-1278 (2016)

${ }^{21}$ J. R. Hendrickson, S. Vangala, N. Nader, K. Leedy, J. Guo, and J. W. Cleary, "Plasmon resonance and perfect light absorption in subwavelength trench arrays etched in gallium-doped zinc oxide film," Applied Physics Letters 107, 191906 (2015)

${ }^{22}$ A. Tang, Z. Mei, Y. Hou, L. Liu, V. Venkatachalapathy, A. Azarov, A. Kuznetsov, and X. Du, "Ga zn-v zn acceptor complex defect in ga-doped zno," SCIENCE CHINA Physics, Mechanics \& Astronomy 61, 77311 (2018)

${ }^{23}$ A.-H. Tang, Z.-X. Mei, Y.-N. Hou, and X.-L. Du, "Photodynamics of gazn-vzn complex defect in ga-doped zno," Chinese Physics B 27, 117802 (2018).

${ }^{24}$ L. J. Brillson and Y. Lu, "Zno schottky barriers and ohmic contacts," Journal of Applied Physics 109, 8 (2011).

${ }^{25} \mathrm{~W}$. Bond, "Measurement of the refractive indices of several crystals," Jour- nal of Applied Physics 36, 1674-1677 (1965).

${ }^{26}$ X. Du, Z. Mei, Z. Liu, Y. Guo, T. Zhang, Y. Hou, Z. Zhang, Q. Xue, and A. Y. Kuznetsov, "Controlled growth of high-quality zno-based films and fabrication of visible-blind and solar-blind ultra-violet detectors," Advanced Materials 21, 4625-4630 (2009).

${ }^{27} \mathrm{R}$. H. Fowler, "The analysis of photoelectric sensitivity curves for clean metals at various temperatures," Physical review 38, 45 (1931).

${ }^{28} \mathrm{~W}$. Li and J. Valentine, "Metamaterial perfect absorber based hot electron photodetection," Nano letters 14, 3510-3514 (2014).

${ }^{29}$ Y. K. Lee, C. H. Jung, J. Park, H. Seo, G. A. Somorjai, and J. Y. Park, "Surface plasmon-driven hot electron flow probed with metal-semiconductor nanodiodes," Nano letters 11, 4251-4255 (2011).

${ }^{30}$ Z. Qi, Y. Zhai, L. Wen, Q Wang, Q Chen, S. Iqbal, G. Chen, J. Xu, and Y. Tu, "Au nanoparticle-decorated silicon pyramids for plasmon-enhanced hot electron near-infrared photodetection," Nanotechnology 28, 275202 (2017).

${ }^{31} \mathrm{~W}$. Lauterborn and T. Kurz, Coherent optics: fundamentals and applications (Springer Science \& Business Media, 2003).

${ }^{32}$ H. Yuan, X. Liu, F. Afshinmanesh, W. Li, G. Xu, J. Sun, B. Lian, A. G. Curto, G. Ye, Y. Hikita, et al., "Polarization-sensitive broadband photodetector using a black phosphorus vertical p-n junction," Nature nanotechnology 10, 707-713 (2015).

${ }^{33}$ Y. Hou, Z. Mei, Z. Liu, H. Liang, C. Gu, and X. Du, "Mgzno based ultraviolet photodetector with high photoresponsivity achieved by fluorine doping," Thin Solid Films 634, 165-168 (2017).

${ }^{34}$ Y. Hou, A. Tang, H. Liang, X. Du, and Z. Mei, "Optical study of a twolevel defect complex in gallium doped zno," Journal of Physics D: Applied Physics 52, 275101 (2019).

${ }^{35}$ O. Katz, G. Bahir, and J. Salzman, "Persistent photocurrent and surface trapping in gan schottky ultraviolet detectors," Applied physics letters $\mathbf{8 4}$ 4092-4094 (2004)

${ }^{36}$ Y. Hou, Z. Mei, Z. Liu, T. Zhang, and X. Du, "Mg0.55zn0.45o solar-blind ultraviolet detector with high photoresponse performance and large internal gain," Applied Physics Letters 98, 103506 (2011)

${ }^{37}$ Y. Hou, Z. Mei, H. Liang, D. Ye, S. Liang, C. Gu, and X. Du, "Comparative study of n-mgzno/p-si ultraviolet-b photodetector performance with different device structures," Applied Physics Letters 98, 263501 (2011).

${ }^{38}$ Y. Hou, Z. Mei, H. Liang, D. Ye, C. Gu, and X. Du, "Dual-band mgzno ultraviolet photodetector integrated with si," Applied Physics Letters 102. 153510 (2013).

${ }^{39}$ Y. Hou, Z. Mei, and X. Du, "Semiconductor ultraviolet photodetectors based on zno and mgxzn1-xo," Journal of Physics D: Applied Physics 47, 283001 (2014) 


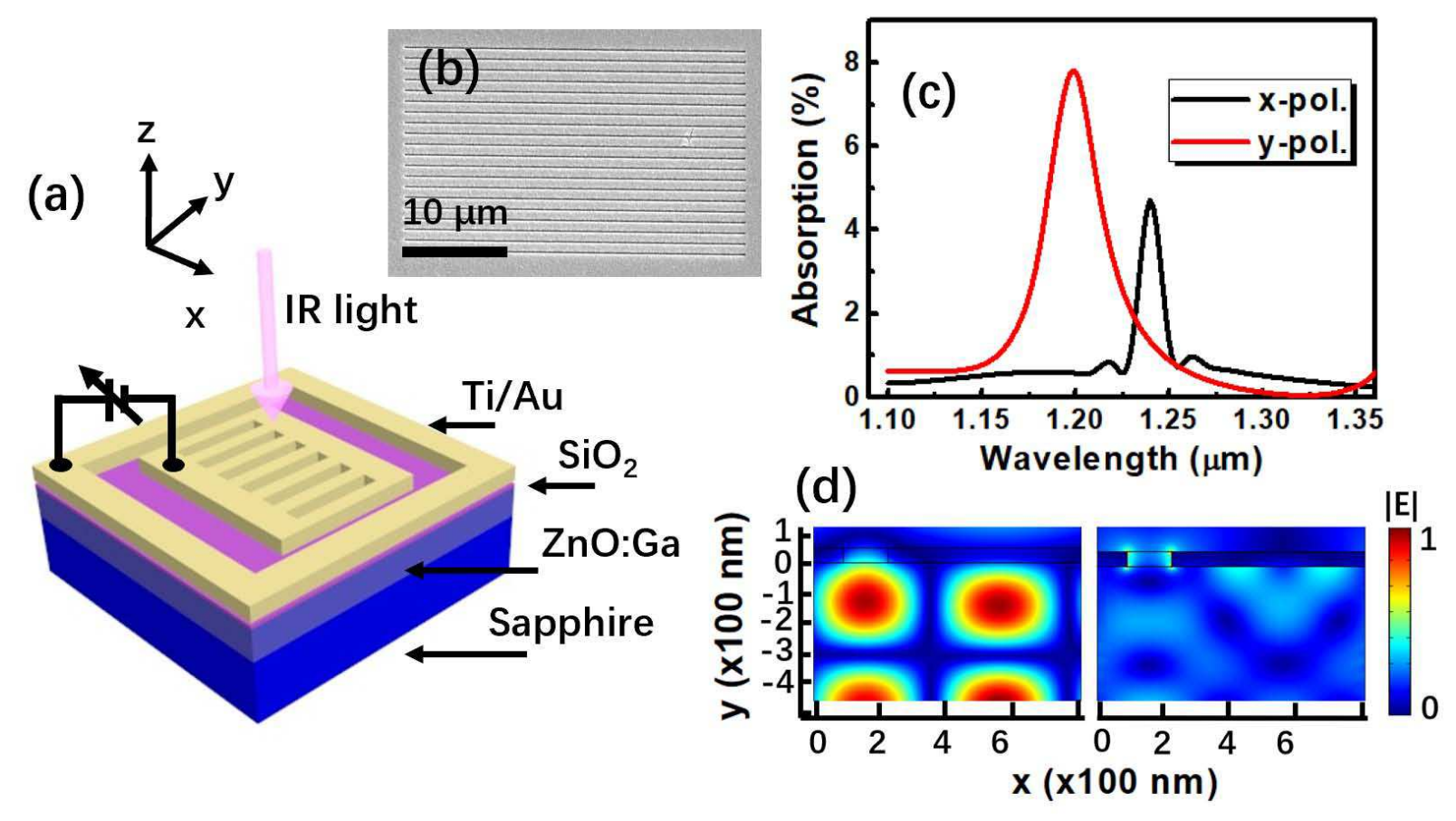




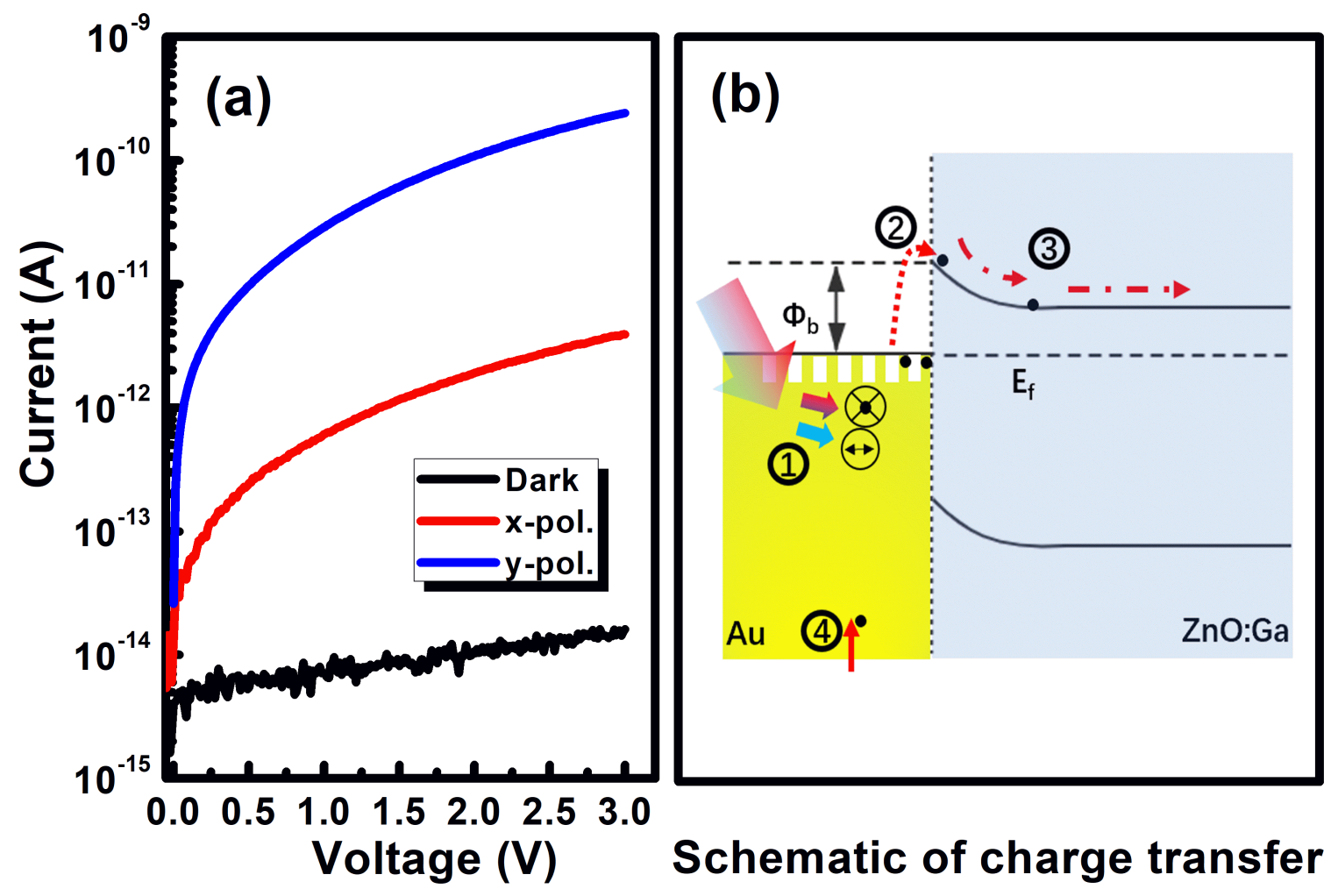



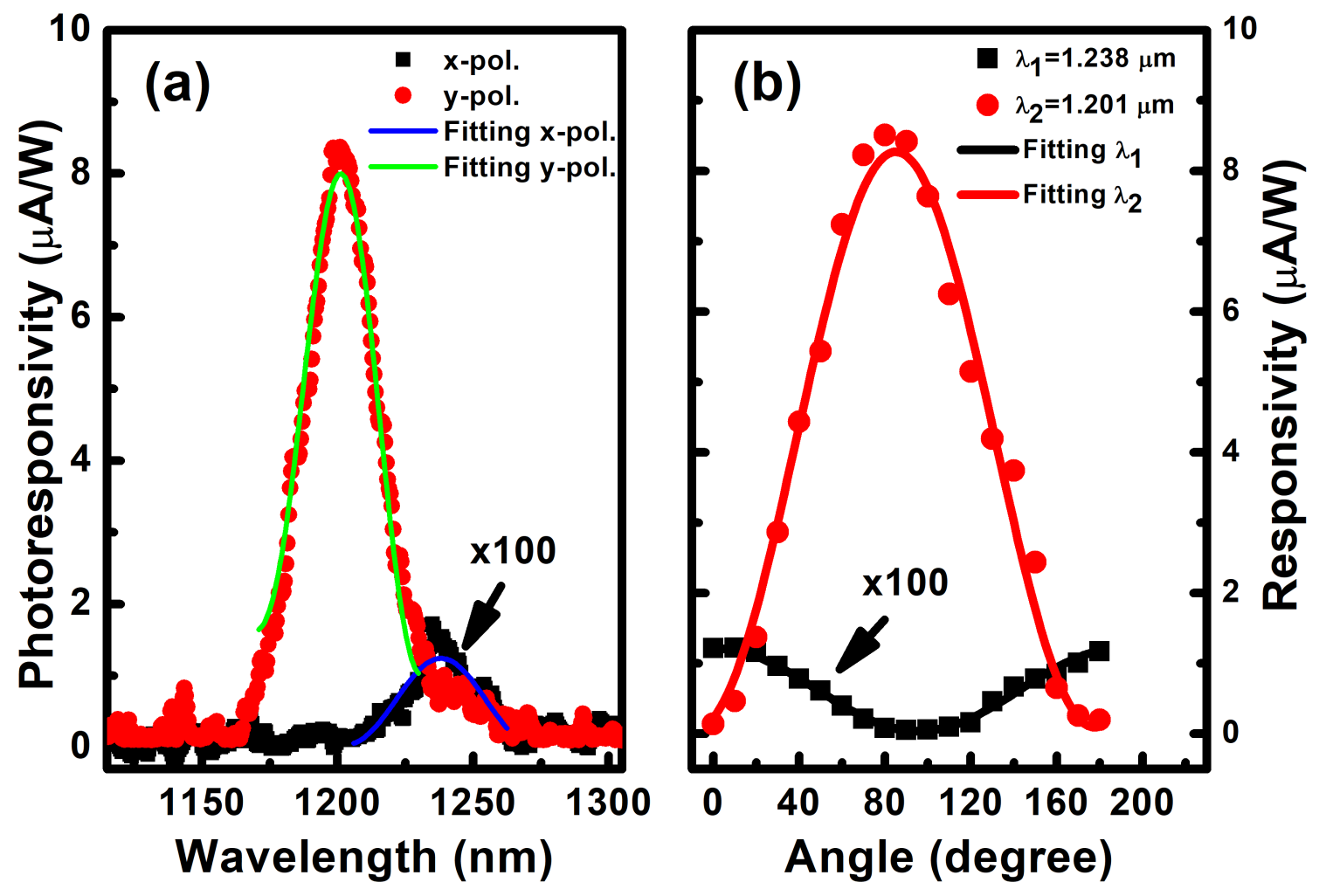


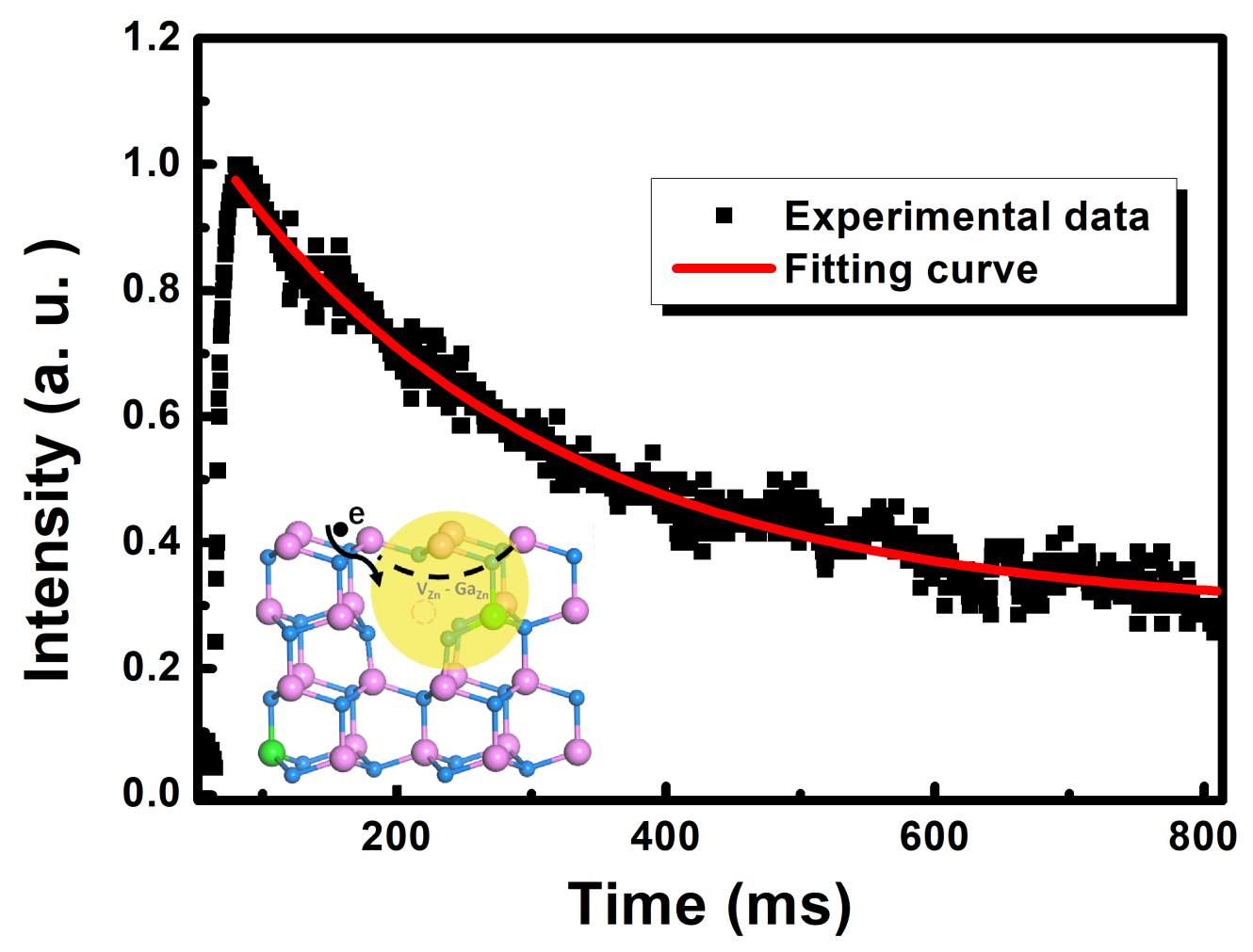



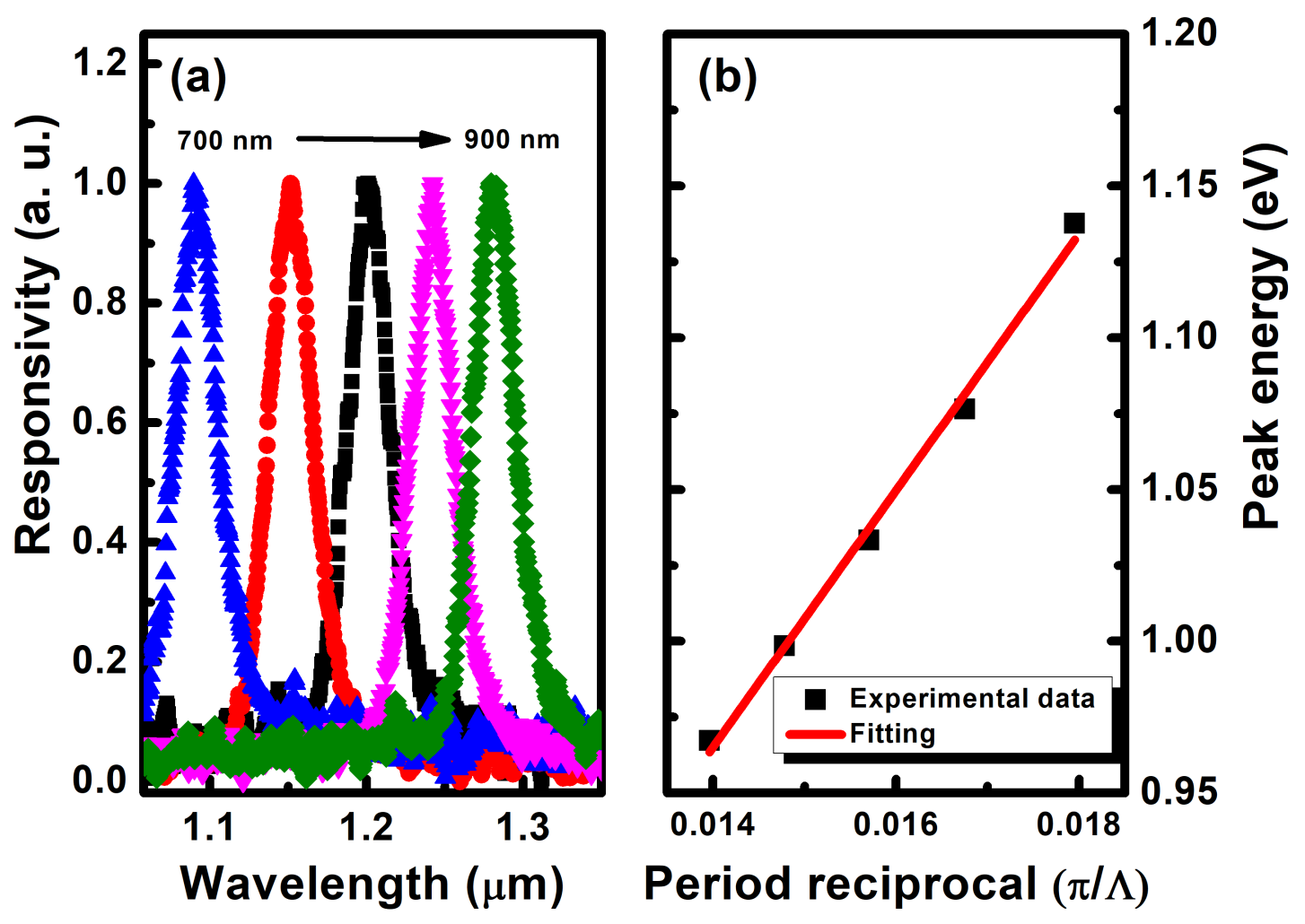